\title{
Low Density Neutrophils Are Increased in Patients With Behçet's Disease but Do 3 Not Explain Differences in Neutrophil Function
}

\section{Graham Wallace ( $\nabla$ g.r.wallace@bham.ac.uk)}

University of Birmingham https://orcid.org/0000-0003-2054-0509

\section{Mariam Murad}

University of Birmingham

Liying Low

University of Birmingham

Matthew Davidson

University of Birmingham

Philip lan Murray

University of Birmingham

Saaeha Rauz

University of Birmingham

\section{Research}

Keywords: Behçet's disease, low density, normal density, neutrophil extracellular traps

Posted Date: May 5th, 2021

DOI: https://doi.org/10.21203/rs.3.rs-481837/v1

License: (c) (i) This work is licensed under a Creative Commons Attribution 4.0 International License.

Read Full License 
3 Low density neutrophils are increased in patients with Behçet's Disease but do

4 not explain differences in neutrophil function.

5

6 Mariam Murad ${ }^{1}$, Liying Low ${ }^{1}$, Matthew Davidson ${ }^{1}$, Philip I Murray ${ }^{1,2}$, Saaeha Rauz ${ }^{1,2}$ Graham

7 R Wallace ${ }^{1,2}$

$8{ }^{1}$ Academic Unit of Ophthalmology, Institute of Inflammation and Ageing, University of

9 Birmingham, United Kingdom; ${ }^{2}$ Birmingham \& Midland Eye Centre, Sandwell and West

10 Birmingham Hospitals NHS Trust, Birmingham, United Kingdom.

ORCID iD: Mariam Murad: 0000-0002-2343-480; Liying Low: 0000-0002-0631-8891; Philip I Murray: 0000-0001-8491-3795; Graham R Wallace: 0000-0003-2054-0509; Saaeha Rauz: 0000-0003-4627-3496.

Corresponding author: Dr Graham R. Wallace Academic Unit of Ophthalmology, Institute of Inflammation and Ageing, University of Birmingham, Edgbaston B15 2 WD United Kingdom. 


\section{Objective}

Behçet's disease (BD) is a multisystem autoinflammatory disease characterised by mucosal ulceration, ocular, neural, joint and skin inflammation. The cause of BD is not known but there is a strong genetic association with HLA-B*51, IL10 and IL23R. Neutrophils are a first line of defence against invading pathogens and have been described as activated in patients with BD. Neutrophils can now be separated into different subsets, such as low density (LDN) and normal density (NDN) that have diverse functional roles. We wished to address neutrophil heterogeneity in patients with BD.

\section{Methods}

Peripheral blood neutrophils were obtained from 32 BD patients and 37 healthy agedmatched controls. Percoll isolation was used to isolate all neutrophils, while Ficol-Hypaque was used to obtain LDN and NDN. Phagocytic capacity and production of reactive oxygen species (ROS), and neutrophil extracellular traps (NET) stimulated with phorbol 12-myristate 13-acetate (PMA) and Escherichia coli (E.coli) were assessed in both groups.

\section{Results}

We have demonstrated reduced phagocytic capacity and ROS production but greater NET production by total neutrophils stimulated with PMA or E.coli from BD patients in comparison with healthy controls. Patients with BD had elevated numbers of LDN and lower number of NDN compared with healthy controls. However, both neutrophil subsets showed the same reduced ROS production and phagocytic function as total neutrophils in both groups.

\section{Conclusion}

Our novel findings indicate that the neutrophil population in BD is heterogeneous and the increased number of LDN in combination with greater NET production may contribute to the inflammatory response and pathogenesis. 
Behçet's disease (BD) is a multisystemic inflammatory disorder characterised by recurrent oral ulcers, genital ulcers, intraocular and neural inflammation skin lesions and thrombotic vascular events (1). The cause of BD remains unknown despite a strong association with HLAB*51, IL10 and IL23RIII2RB [2] The prevalence rate of BD in the UK is 14.61 (95\% Cl 13.3515.88) per 100,000 population. [3] Between $50-90 \%$ of BD patients have intraocular involvement manifestations, such as recurrent, non-granulomatous panuveitis, hypopyon and retinal vasculitis. Posterior segment inflammation of the eye also involves occlusion of retinal veins and later occlusion of the retinal arterial circulation that can lead to irreversible blindness. (4).

Neutrophils are a major group of the immune cell population that are present in a resting state in the circulation of healthy individuals and play a dynamic role within an inflammatory response. Neutrophils are phagocytic cells that ingest infectious agents, such as bacteria and fungi, that are destroyed by the production of reactive oxygen and nitrogen species (ROS and NOS) known as oxidative burst, with the release of neutrophil granule components and the production of elastase (5). The build-up of oxidative stress and the production of oxygen radicals may cause DNA damage, oxidation of lipids, lipoproteins and proteins and can be linked to mutations in immunoglobulins that have been implicated in the formation of inflammatory diseases (6). Neutrophil extracellular traps (NET) are web-like structures composed of cytosolic and granule proteins of decondensed chromatin, produced to protect against infection by pathogens (7). NET proteins are derived from primary granules (neutrophil elastase and myeloperoxidase), secondary granules (lactoferrin and pentraxin 3) and tertiary granules (matrix metalloproteinase). NET formed by activated neutrophils trap bacteria and other pathogens leading to their destruction (8).

Neutrophil hyperfunction, in particular increased neutrophil chemotaxis, has been linked with BD for over 40 years (9). Neutrophils have been reported in biopsies of active oral and genital ulcers, the skin lesions of erythema nodosum and the skin pathergy test $(10,11)$. Neutrophils from HLA-B*51-positive patients showed an increase in the chemotactic response toward several stimuli suggesting that the HLA region can exert a regulatory control on PMN functions. $(12,13) \gamma \delta$ T cells and monocytes activated via Toll-like receptor-2 (TLR2) have been 
implicated in releasing neutrophil stimulating molecules that induce chemotaxis in neutrophils from patients with BD and healthy controls in response to several stimuli. (14) Neutrophils from BD patients display elevated superoxide production and increased lysosymal enzyme production in response to different stimuli $[15,16]$. Recent studies have shown increased NET production by neutrophils from patients with BD. $[17,18]$ Increased expression of CD11b possibly as a result of the interaction of neutrophils with activated platelets, enhanced platelet-neutrophil aggregate formation. [19]. The neutrophillymphocyte ratio was significantly higher in patients with active BD, although not significantly different between patients with or without thrombosis. [20] Certain patients with BD can be successfully treated with colchicine which is a neutrophil inhibitor. [21,22]

Neutrophils were considered to be a homogenous population of differentiated cells with a distinct and conserved function. However, increasing evidence has demonstrated a phenotypic heterogeneity and functional flexibility of the neutrophil population. Low density neutrophils (LDN) are banded and appear as myelocyte-like cells [23]. LDN have been classified as immature cells based on morphology and gene expression, although surface markers (CD66b, CD11b) suggest a mature phenotype. LDN can be subdivided into those with a pro-inflammatory phenotype and function and those who have been classified as granulocytic myeloid derived suppressive (G-MDSC) with an immunosuppressive phenotype and function. [24,25] Normal density neutrophils (NDN) separate with the red-blood cell layer in a Ficoll gradient and are described as a homogenous population of resting neutrophils [24].

In this paper we have assessed neutrophil phenotype and function in patients with BD. The results show increased LDN and NET production but decreased production of ROS and phagocytosis in disease cohort compared to controls. 
Peripheral blood was obtained from 32 patients with BD attending the Birmingham National Centre of Excellence for BD. All patients fulfilled the 1990 International Study Group criteria for BD. [26] Age matched healthy control samples were obtained from University of Birmingham laboratory staff $(n=37)$. Informed written consent was obtained. The research followed the tenets of the Declaration of Helsinki and was approved by the NHS Research Ethics Committee (LREC ref: 08/H1206/165).

Total Neutrophil isolation.

Peripheral venous blood samples were collected in a micro vacutainer containing heparin and processed within 4 hours. Under sterile conditions the samples were dispensed into a $50 \mathrm{ml}$ Falcon ${ }^{\circledR}$ tubes and $2 \%$ dextran added at a ratio of $1 \mathrm{ml}$ dextran: $6 \mathrm{ml}$ blood and the tubes inverted 5 times. The Falcon ${ }^{\circledR}$ tubes with a loosened lid were left in the hood for 30-40 minutes, for yellow coloured buffy coat to appear. $5 \mathrm{mls}$ of $56 \%$, Percoll was added into a $15 \mathrm{ml}$ Falcon ${ }^{\circledR}$ tube, then $2.5 \mathrm{ml}$ of $80 \%$ Percoll was underneath to form a gradient. Buffy coats were added to the gradients and centrifuged at $1100 \mathrm{rpm}$ for 20 minutes at room temperature with one acceleration and zero de-acceleration. Two distinct layers of cells on the gradients were observed. The top layer represented the peripheral blood mononuclear cells (PBMC) was discarded. The second layer containing neutrophils were collected and placed into a new $15 \mathrm{ml}$ Falcon ${ }^{\circledR}$ tube containing RPMI 1640 medium + foetal calf serum (10\% FCS) + L-GlutaminePenicillin-Streptomycin solution (1\% L-GPS) (completed medium; CM) and centrifuged or 10 minutes at $1600 \mathrm{rpm}$ at room temperature with one acceleration and zero de-acceleration. All neutrophils were isolated (purified) using the EasySep Human Neutrophil Enrichment Kit, according to the manufacturer's instructions, and re-suspended in $\mathrm{CM}$.

Blood samples were collected in a micro vacutainer containing heparin and processed within 4 hours. The Ficoll-Hypaque (FH) solution under sterile conditions was placed into a $50 \mathrm{ml}$ Falcon ${ }^{\circledR}$ tube in the ratio of $2 \mathrm{ml}$ of $\mathrm{FH}: 1 \mathrm{ml}$ of blood. The diluted blood was slowly layered over the $\mathrm{FH}$ solution and centrifuged for 40 minutes at $400 \mathrm{~g}$ at $22^{\circ} \mathrm{C}$ with 0 break. After 
centrifugation the LDN, located at the interface between plasma and FH layers (PBMC), were removed. The cells were transferred to a $15 \mathrm{ml}$ Falcon ${ }^{\circledR}$ tube containing $10 \mathrm{ml}$ of PBS and centrifuged for 10 minutes at $400 \mathrm{~g}$ at $4^{\circ} \mathrm{C}$. The supernatant was discarded, the wash repeated and the cells were used as required. NDN, granulocyte layer, located above the FH were removed and $100 \mu \mathrm{l}$ of the cell suspension was transferred to $15 \mathrm{ml}$ Falcon ${ }^{\circledR}$ tube. The cell suspension was lysed with red blood lysis buffer in the ratio of $100 \mathrm{ul}: 1 \mathrm{ml}$, vortexed and incubated in the dark for 20 minutes. The cells were centrifuged at $250 \mathrm{~g}$ for 5 minutes at room temperature, the supernatant was discarded and NDN were purified using the EasySep Human Neutrophil Enrichment Kit, according to manufactures instructions.

Cell viability and purity.

Viability of neutrophil populations was assessed by Trypan Blue staining. To determine purity cells were re-suspended in $50 \mu \mathrm{l}$ of $\mathrm{CM}$, slides constructed and $50 \mu \mathrm{l}$ of the sample was added into the funnel and the samples were spun in a cytospin centrifuge at $300 \mathrm{rpm}$ for $10 \mathrm{~min}$. Post spinning, the slide was left to air dry for $30 \mathrm{~min}$, dipped into cold methanol for 30 secs and left to air dry for $10 \mathrm{~min}$. A small drop of diluted Giemsa (1/20) was added onto the cells and the slide was further left for $30 \mathrm{~min}$. The slide was rinsed thoroughly with distilled water and left to air dry for 1 hour. The slide was visualised under a light microscope using X200 magnification

Neutrophil phagocytosis and oxidative burst in whole blood samples

A quantitative analysis of neutrophil phagocytosis and oxidative burst activity in whole blood samples was quantified using Phagotest and Phagoburst kits (Glycotope Biotechnology).

Generation of cell free DNA (cfDNA).

In this paper cfDNA will be used as a marker of NET production. Isolated neutrophils $\left(2 \times 10^{5}\right.$ cells $/ \mathrm{ml}$ ) were seeded $(200 \mu \mathrm{l})$ into wells of clear flat bottomed 96 well plates and stimulated with 25nM PMA (diluted from stock solutions with $\mathrm{CM}$ for 3 hours at $37^{\circ} \mathrm{C}$ and $5 \% \mathrm{CO}_{2}$ atmosphere. After incubation the supernatant was removed, transferred into 600ul Eppendorf tube and centrifuged at $2200 \mathrm{~g}$ for 10 minutes at $4^{\circ} \mathrm{C} .100 \mathrm{ul}$ of cell free supernatant was removed and placed into black 96 well plates and incubated with 1uM SYTOX Green Dye for 10 minutes in the dark at room temperature. The florescence was measured using BioTek 
Synergy 2 fluorometric plate reader with excitation at $485 \mathrm{~nm}$ and emission at $528 \mathrm{~nm}$. All samples were analysed in duplicate. A calibration step was performed using the cell free supernatant from unstimulated neutrophils and buffer controls were analysed in duplicate. $\lambda$ DNA $(0.3 \mu \mathrm{g} / \mu \mathrm{l}$ was diluted $1: 20$ in PBS) was used to calibrate the samples with a standard curve ranging from $0-1000 \mathrm{ng} / \mathrm{ml}$. The top standard was serially diluted 7 times with a final dilution of 1:15 with 1uM SYTOX Green Dye. The same assay was followed using opsonised E.coli $\left(1-2 \times 10^{9}\right.$ bacteria per $\left.\mathrm{ml}\right)$.

\section{Visualisation of cfDNA}

Purified neutrophils ( $2 \times 10^{5}$ cells $/ \mathrm{ml}$ ) were re-suspended in $2 \mathrm{ml}$ of $\mathrm{CM}$ and seeded onto 8 well chamber slides and incubated for 30 minutes at $37^{\circ} \mathrm{C}$ in a $5 \% \mathrm{CO}_{2}$ atmosphere to allow cell adherence. After 30 minutes incubation neutrophils were stimulated with 25nM PMA for 3 hours at $37^{\circ} \mathrm{C}$ in a $5 \% \mathrm{CO}_{2}$. All agonists were prepared using the stock solutions in $\mathrm{CM}$ which also served as a buffer control. Post-stimulation, cells were fixed with $4 \%$ paraformaldehyde (PFA) $(200 \mu \mathrm{l})$ and incubated for 30 minutes at $37^{\circ} \mathrm{C}$ in $5 \% \mathrm{CO}_{2}$. Post fixation, the slides were washed in sterile phosphate-buffered saline (PBS) 3 times, once with $0.1 \%$ Triton X-100 for 1 minute and finally a 5-minute wash in sterile PBS, all at room temperature. Cells were incubated (5 minutes) with $1 \mu \mathrm{M}$ SYTOX green Dye at room temperature followed by a 5minute wash in sterile PBS at room temperature. The slides were then mounted in fluoromount medium and were imaged using a LEICA DMI 6000B microscope at $x 10, x 20$ and x40 objective. The same assay was followed using opsonised E.coli (1-2 x $10^{9}$ bacteria per $\left.\mathrm{ml}\right)$.

\section{Statistical analysis}

The data was statistically analysed using the Kruskal-Wallis test and Dunn's Multiple Comparison Test (Post-Hoc) for a comparison between 3 different groups, and a MannWhitney $\mathrm{U}$ test was performed for a comparison between 2 different groups using GraphPad Prism version 5.03. All $p$ values were considered significant at $p<0.05$. The ratio of NET was calculated by using the following formula;

Stimulated (PMA/E.coli)

Unstimulated (PMA/E.coli) 
Patients with BD (49 $\pm 4(\%) n=10)$ had a significantly reduced phagocytic activity of neutrophils compared with healthy individuals ( $88 \pm 2$ (\%) $n=17$ ) (Fig 1A). Production of ROS was assessed by stimulating neutrophils with opsonised E.coli in BD patients ( $52 \pm 5$ (\%) $n=10)$ produced a reduced amount of reactive oxygen species in comparison to age-matched healthy volunteers $(80 \pm 4(\%) n=16)$ (Fig 1B). Neutrophils were stimulated with PMA to determine release of cell-free DNA. Unstimulated neutrophils from patients with BD $(58 \pm 2$ (ng/ml) $n=11$ ) showed significantly greater spontaneous cell free DNA release compared to unstimulated cells from healthy controls $(31 \pm 2(\mathrm{ng} / \mathrm{ml}) \mathrm{n}=10)$. PMA induced significantly greater cfDNA release by neutrophils both from patients with BD $(110 \pm 2(\mathrm{ng} / \mathrm{ml})$ vs $58 \pm 2$ $(\mathrm{ng} / \mathrm{ml}) \mathrm{n}=11)$ and healthy controls $(60 \pm 5(\mathrm{ng} / \mathrm{ml})$ vs $31 \pm 2(\mathrm{ng} / \mathrm{ml}))$ compared with unstimulated cells. However, neutrophils from patients with BD $(110 \pm 2(\mathrm{ng} / \mathrm{ml})$ vs $60 \pm 5$ $(\mathrm{ng} / \mathrm{ml}))$ responded to stimulus with significantly greater cfDNA release than cells from healthy controls (Fig. 2A).

In a second analysis spontaneous release of cfDNA was greater in unstimulated neutrophils from healthy controls $(57 \pm 1(\mathrm{ng} / \mathrm{ml}))$ compared with BD patients $(51 \pm 5(\mathrm{ng} / \mathrm{ml}))$. Stimulation with opsonised E.coli significantly increased cfDNA release from healthy controls $115 \pm 5$ $(\mathrm{ng} / \mathrm{ml})$ vs $57 \pm 1(\mathrm{ng} / \mathrm{ml})$ and BD patients $84 \pm 1(\mathrm{ng} / \mathrm{ml})$ vs $51.3 \pm 1(\mathrm{ng} / \mathrm{ml})$, however in comparison to PMA stimulation neutrophils from healthy controls produced significantly more than stimulated neutrophils from patients with BD (115 $\pm 1(\mathrm{ng} / \mathrm{ml})$ vs $84 \pm 1(\mathrm{ng} / \mathrm{ml}))$ (Fig. 2B). A comparison of PMA and E.coli stimulation is shown in Fig. 2C

In order to visualise NET in stimulated neutrophils with PMA and E.coli, Sytox Green was used. The results were comparable to results obtained for the productions of cf DNA by all neutrophils from patients with BD compared with healthy controls. Figure 3 showed DNA strands i.e. NET formation in neutrophils stimulated with PMA or E.coli from patients with BD (Fig. 3B and D).

To address whether the differences in ROS, cfDNA and phagocytosis were due to different subsets of cells, LDN and NDN were prepared on Ficoll gradients. Morphologically, LDN have a curved nucleus with two or fewer nuclear lobes while NDN show mature segmented nuclei. 
Patients with BD showed an increased LDN $(6 \pm 0.4$ vs $2 \pm 0.8(n=11))$ and reduced NDN $(2 \pm$ 0.7 vs $6 \pm 0.3(\%)(n=11)$ ) count compared with healthy controls ( $n=12)$ (Fig. 4).

221

222

223

224

Whether these populations could account for the differences seen in total neutrophils was considered and LDN and NDN were assessed as above. The results showed a statistically significantly lower phagocytic capacity of both LDN (28 \pm 5 (\%) ( $n=12)$ vs $98 \pm 1$ (\%) (n=11) and NDN (28 $\pm 5(n=12)$ vs $96 \pm 3(\%)(n=11)$ in BD patients compared with healthy controls (Fig. 5A), and a similar decrease in ROS production (32 \pm 18 vs $96 \pm 3$ (\%)) (32 \pm 6 vs $91 \pm 14$ (\%) (Fig.5B). Spontaneous cfDNA production by both LDN (49 \pm 3 vs $29 \pm 1$ ) NDN (44 \pm 8 vs 31 \pm 1 ) subsets were significantly greater in patients with BD compared with healthy controls (Figs.6A and 7A).

Figure 6A shows that cfDNA taken from cultures of PMA stimulated LDN of patients diagnosed with BD was not only significantly higher (128 \pm 6 vs $49 \pm 10(\%)$ ) than cell free DNA content taken from unstimulated BD cell cultures but was also significantly higher (128 \pm 6 vs $35 \pm 8(\%)$ ) in comparison to cell free DNA taken from cultures of PMA stimulated neutrophils of healthy individuals (Fig. 6A). The results showed PMA stimulated LDN of healthy controls was significantly ( $35 \pm 8$ vs $30 \pm 2$ (\%)) higher compared with unstimulated LDN in healthy individuals.

The cfDNA taken from cultures of PMA stimulated NDN of BD was not only significantly higher (99 \pm 6 vs $44 \pm 8(\%)$ ) than cfDNA content taken from unstimulated BD neutrophil cultures but was also significantly higher ( $99 \pm 6$ vs $55 \pm 8(\%)$ ) in comparison to cfDNA taken from cultures of PMA stimulated neutrophils of healthy individuals (Fig.7A). The production of cfDNA from cultures of primed NDN of healthy individuals was also significantly (55 \pm 8 vs $31 \pm 1$ (\%)) enhanced (Fig. 7A). An increase ( $44 \pm 8$ vs $31 \pm 1(\%)$ ) in spontaneous cfDNA was observed in NDN of BD patients in contrast with healthy individuals (Fig. 7A).

While PMA is commonly used to investigate NET production, it is a non-physiological stimulus. To address this total neutrophils, NDN and LDN subsets were stimulated with E.coli under the same conditions. Stimulation with E.coli showed a significantly reduced production of NETs. Figure $6 \mathrm{~B}$ showed that cfDNA obtained from cultures of E.coli stimulated LDN of BD patients was significantly lower (67 \pm 5 vs $82 \pm 3(\%)$ ) than cfDNA content taken from E.coli stimulated neutrophils of healthy individuals; despite being higher (67 \pm 5 vs $49 \pm 4$ (\%)) compared with 
unstimulated LDN of BD patients. The results also showed a significant ( $82 \pm 3$ vs $55 \pm 3(\%)$ ) increase in the production of cfDNA by stimulated LDN compared with unstimulated LDN in

251 healthy individuals. The results showed that unstimulated LDN cell cultures produced higher amount (55 \pm 3 vs $49 \pm 3(\%)$ ) of spontaneous cfDNA compared with unstimulated LDN cell cultures of BD patients (Fig. 6B).

254 Stimulation with E.coli showed a significantly reduced production of NET in stimulated (65 \pm 2559 vs $79 \pm 1(\%)$ ) neutrophil cell cultures of BD patients compared with healthy controls (Fig.7B). A significant increase (65 \pm 9 vs $50 \pm 4(\%)$ ), of stimulated E.coli neutrophil BD cell cultures compared with unstimulated was observed. A similar pattern of a significant increase $(79 \pm 1$ vs $54 \pm 3(\%))$ of cfDNA in stimulated neutrophil cell cultures obtained from healthy controls compared with unstimulated. Figure 7B showed significantly reduced spontaneous cfDNA in in healthy individuals and BD patients ( $50 \pm 4$ vs $53 \pm 2(\%)$ ).

261 The results showed the control group (unstimulated cells) for PMA stimulated LDN and NDN of healthy individuals produced less spontaneous cfDNA compared with the control group for E.coli stimulated LDN and NDN of healthy individuals compared with patients with BD (Fig. 6A, 6B, 7A, 7B). However, NET in LDN and NDN with the stimulant E.coli was reduced in patients with BD patients compared with healthy controls (Fig. 6B, 7B). 
Neutrophils have long been implicated in the pathogenesis of BD. Recent advances in neutrophil subsets and functions allowed us to reanalyse this relationship. In our cohort of patients with $\mathrm{BD}$, total neutrophils have reduced phagocytic capacity and ROS production when compared with age matched healthy controls. By comparison, these cells produce more cfDNA in response to stimuli, PMA and E.coli. Patients with BD had greater numbers of LDN compared with healthy controls, but both LDN and NDN showed a similar functional profile as total neutrophils. Although LDN are increased in patients with BD this does not explain the functional differences between patients and healthy controls.

With regards to ROS production and phagocytosis these results differ from previous reports. Gogus et al. showed significantly higher oxidative burst by neutrophils in patients with BD compared with $\mathrm{HC}$ in response to monosodium urate crystals. (15) Perazzio et al. found no significant differences between BD patients and controls with regard to oxidative burst, phagocytic activity, microbicide activity or cytokine production. However, the cells from patients with severe BD exhibited significantly higher oxidative burst activity, both before and after PMA stimulation, compared with cells from patients with mild BD. (27) These differences may be due to the heterogeneity of the patient cohorts, treatment regimens or the effects of the type of stimuli used. In the current studies highly purified neutrophils were used that may have influenced the results. Understanding ROS production is important as neutrophil derived ROS induces carbonylation of fibrinogen leading to increased polymerisation and protection from lysis. Neutrophils from patients with BD were more effective at modifying fibrinogen a process that is involved in clot formation and thrombosis, that may be related to vasculitis in BD. $(28,29)$

Reduced phagocytic function has been reported during the first 24 hours in patients with sepsis was associated with reduced survival. This may represent a neutrophil deactivation state or unresponsiveness due to persistent stimulation by cytokines. Similarly, impaired neutrophil phagocytic function and ROS production was described in patients with antineutrophil cytoplasmic antibody associated vasculitis a condition characterised by autoimmune small vessel inflammation. [30] In patients with SLE while ROS production was 
oxidative damage than the inactive group. [31] These findings may be relevant in conditions with a constant inflammatory response with continual stimulation of neutrophils, and restimulation of such neutrophils results in a reduced production of functional activity. Although it remains to be clarified, there is no indication that the reduced function of neutrophils from patients with $B D$ is due to the immunosuppressive drugs regimens used to control their condition. One possible mechanism for reduced PMN function in BD could be sleep deprivation.

We have shown for the first time that patients with BD display neutrophil heterogeneity with a high LDN and low NDN count compared with healthy controls. Both LDN and NDN subsets showed reduced ROS production and phagocytic function indicating that difference in numbers of these subsets was not responsible for this phenotype of total neutrophils of patients with BD. These findings were comparable to studies that showed increased levels of LDN in patients with SLE. [32] Moreover, LDN from patients with SLE showed a similar immature phenotype as observed in this study. [33] Recent studies have reported reduced production of ROS and anti-tumour properties by LDN in cancer patients. [34] The presence of LDN may have an important role in the persistent inflammation characteristic and immune response induced in $\mathrm{BD}$ patients. A recent review discusses further differences between neutrophil phenotype in different disease with energy metabolism and glycolysis driving ROS and NET production in RA, while decreased redox reactions drive the same in SLE. [35]

NET formation has an important role in the development and preservation of autoimmune diseases, organ damage in chronic inflammatory disorders and cancer. [36-38] In our study total neutrophils, LDN and NDN in patients with BD showed increased production of cfDNA compared with healthy controls when stimulated with PMA or E.coli. NET can be considered to form part of the host defence system during BD. Inadequate elimination of pathogens due to the reduced phagocytosis and ROS production contributes towards defective killing of bacteria at mucosal surfaces in patients with $\mathrm{BD}$ may contribute to the persistent inflammation characteristic of these patients. In a study of Middle Eastern patients with BD PMN from patients released significantly more NET compared to controls. This was linked to expression of higher levels of PAD4, and NET production was reduced using a PAD4 inhibitor. Serum from patients stimulated PMN from healthy controls to produce more NET. NETs were identified around blood vessels in tissue sections from patients with BD linking NET to 
vasculitis. Moreover, NET from patients with BD decreased proliferation and increased apoptosis when cultured with endothelial cells. [17] Le Joncour et al. described increased serum cfDNA and MPO-DNA complexes in patients with active BD compared with inactive patients and healthy controls, and in patients with vasculitis compared with those without. Plasma from patients with BD induced greater thrombin generation a response correlated with cfDNA and MPO-DNA levels. In support of Safi et al. NETs were identified in areas of vasculitis in biopsy specimens. [18] Patients with both active and inactive BD had Increased levels of SCD4OL in their plasma, that induced ROS and NET production in neutrophils, and increased expression of Mac-1 that together could contribute to binding to vasculature. [39]

While plasma from patients with BD drives NET production, saliva does not. Saliva of healthy controls, collected in the morning, induced neutrophils to released NET independent of elastase or NADPH. These NET had a greater capacity to kill bacteria and were more resistant to DNAase. By comparison, saliva from patients with aphthous ulcers or BD did not induce NET production due for different reasons. In patients with aphthous ulcers sialyl Lewis $\mathrm{X}$, a molecule driving NET production was lost from salivary mucins. This was not this case in BD as some unknown factor in saliva was inhibiting the response. [40] The possibility that these responses were influenced by altered oral microbiome which is different in patients with aphthous or BD ulceration is intriguing. [41,42] Likewise the potential influence of other microbiomes at sites of mucosal inflammation in patients with $B D$ on immune responses should be considered.

The results suggested that enhanced production of NET in BD patients may contribute towards the pathology of BD. However, to date most data has utilised imaging cfDNA and it is possible that in inflammatory and necrotic sites this is not all derived from neutrophils. [43] More detailed identification of the components of NET, including histone $A 3$, myeloperoxidase and elastase should be undertaken. [44] Moreover, challenging neutrophils with more physiological stimuli is required. In murine studies PAD4 is not required NET production in all situations and responses to LPS from different sources varies substantially. $[45,46]$ Although the pathogenesis of BD remains unclear, persistent mucosal ulceration driving a dysfunctional inflammatory response could lead to an increase in neutrophil:lymphocyte ratio, LDN and enhanced NET release and subsequent endothelial activation and vasculitis. While inflammation is treated in patients with BD these results 
360

361

362

363

364

365

366

367

368

369

370

371

372

373

374

375

376

377

378

379

380

381

382

383

suggest NET production by neutrophils is a novel, attractive therapeutic target in patients with BD. [47]

There are several limitations to this study. Firstly, the different analyses were not performed on all the patients or controls due to the requirement to use neutrophils within a few hours of collection. Further studies should analyse phagocytosis, ROS and NET production in the same cells. Secondly patients with BD have different manifestations of the conditions and were on different treatments and this should be addressed in future studies. Finally, as discussed above LDN are not a homogenous, stable population and further investigation of such diversity in BD is needed both in blood and tissue. (48)

In summary, this study may indicate towards a dysfunctional phenotype and function displayed by neutrophils in BD. Taken together the data suggests that in BD persistent inflammation drive release of LDN from the bone marrow. The systemic environment influences both LDN and NDN to produce the phenotype described, that in turn drives mucosal inflammation and vascular damage.

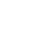

7 
(1) Tong B, Liu X, Xiao J, Su G· Immunopathogenesis of Behcet's Disease. Front Immunol. 2019 10:665. doi: 10.3389/fimmu.2019.00665. eCollection 2019.

(2) Thomas T, Chandan JS, Subramanian A, et al. Epidemiology, morbidity and mortality in Behçet's disease: a cohort study using The Health Improvement (THIN). Rheumatology (Oxford). 2020; 59:2785-2795. doi:10.1093/rheumatology/keaa010

(3) Morton LT, Situnayake D, Wallace GR. Genetics of Behçet's disease Curr Opin Rheumatol 2016; 28:39-44. doi: 10.1097/BOR.0000000000000234.

(4) Cunningham ET Jr, Tugal-Tutkun I, Khairallah $M$, et al Behçet Uveitis. Ocul Immunol Inflamm. 2017; 25:2-6. doi: 10.1080/09273948.2017

(5) Wright HL, Moots RJ, Bucknall RC, Edwards SW. Neutrophil function in inflammation and inflammatory diseases. Rheumatology (Oxford). 2010; 49:1618-31. doi: 10.1093/rheumatology/keq045

(6) Winterbourn CC, Kettle AJ, Hampton MB. Reactive oxygen species and neutrophil function. Ann Rev Biochem. 2016; 85:765-92. doi: 10.1146/annurev-biochem-060815014442.

(7) Papayannopoulos V. Neutrophil extracellular traps in immunity and disease. Nat Rev Immunol. 2018; 18:134-147. doi: 10.1038/nri.2017.105

(8) Castanheira FVS, Kubes P. Neutrophils and NETs in modulating acute and chronic inflammation. Blood. 2019; 133:2178-2185. doi: 10.1182/blood-2018-11-844530.

(9) Matsumura N, Mizushima Y. Leucocyte movement and colchicine treatment in Behcet's disease. Lancet. 1975; 2(7939):813. doi: 10.1016/s0140-6736(75)80031-6.

(10) Jorizzo JL, Abernethy JL, White WL, et al Mucocutaneous criteria for the diagnosis of Behçet's disease: an analysis of clinicopathologic data from multiple international centers. J Am Acad Dermatol. 1995; 32:968-76 doi: 10.1016/0190-9622(95)91333-5

(11) Ergun T, Gürbüz O, Dogusoy G, et al Histopathologic features of the spontaneous pustular lesions of Behçet's syndrome. Int J Dermatol. 1998; 37:194-196 doi: 10.1046/j.1365-4362.1998.00276.x

(12) Sensi A, Gavioli R, Spisani S, et al et al HLA B51 antigen associated with neutrophil hyper-reactivity. Dis Markers. 1991; 9:327-331.

(13) Neves FS, Carrasco S, Goldenstein-Schainberg C, et al Neutrophil hyperchemotaxis in Behçet's disease: a possible role for monocytes orchestrating bacterial-induced innate immune responses. Clin Rheumatol. 2009; 28:1403-10. doi: 10.1007/s10067-009-12611265.

(14) Yamashita N, Kaneoka H, Kaneko S, et al Role of gammadelta T lymphocytes in the development of Behçet's disease. Clin Exp Immunol. 1997; 107:241-247 doi: 10.1111/j.1365-2249.1997.274-ce1159.x

(15) Gogus F, Fresko I, Elbir Y, Eksioglu-Demiralp E, Direskeneli H. Oxidative burst response to monosodium urate crystals in patients with Behçet's syndrome. Clin Exp Rheumatol. 2005; 23:S81-85.

(16) Eksioglu-Demiralp E, Direskeneli H, Kibaroglu A, et al Neutrophil activation in Behçet's disease. Clin Exp Rheumatol. 2001; 19:S19-24.

(17) Safi R, Kallas R, Bardawil T, et al Neutrophils contribute to vasculitis by increased release of neutrophil extracellular traps in Behçet's disease. J Dermatol Sci. 2018; 92:143150. doi: 10.1016/j.jdermsci.2018.08.010 
(18) Le Joncour A, Martos R, Loyau S, et al Critical role of neutrophil extracellular traps (NETs) in patients with Behcet's disease. Ann Rheum Dis. 2019 78:1274-1282. doi: 10.1136/annrheumdis-2018-214335

(19) Macey M, Hagi-Pavli E,Stewart J, et al Age, gender and disease-related platelet and neutrophil activation ex vivo in whole blood samples from patients with Behçet's disease. Rheumatology (Oxford). 2011; 50:1849-59. doi: 10.1093/rheumatology/ker17

(20) Avci A, Avci D, Erden F, et al Can we use the neutrophil-to-lymphocyte ratio, plateletto-lymphocyte ratio, and mean platelet volume values for the diagnosis of anterior uveitis in patients with_Behcet's_disease? Ther Clin Risk Manag. 2017; 13:881-886. doi: 10.2147/TCRM.S135260

(21) Saleh Z, Arayssi T. Update on the therapy of Behçet disease. Ther Adv Chronic Dis. 2014; 5:112-34. doi: 10.1177/2040622314523062

(22) Park UC, Kim TW, Yu HG. Immunopathogenesis of ocular Behçet's disease. J Immunol Res. 2014; 2014:653539. doi: 10.1155/2014/653539. Epub 2014 Jul 2.

(23) Wright HL, Makki FA, Moots RJ, Edwards SW. Low-density granulocytes: functionally distinct, immature neutrophils in rheumatoid arthritis with altered properties and defective TNF signalling. J Leukoc Biol. 2017; 101:599-611. doi: 10.1189/jlb.5A0116-022R.

(24) Scapini P, Marini O, Tecchio C, Cassatella MA. Human neutrophils in the saga of cellular heterogeneity: insights and open questions. Immunol Rev. 2016; 273:48-60. doi: 10.1111/imr.12448

(25) Silvestre-Roig C, Fridlender ZG, Glogauer M, Scapini P. Neutrophil diversity in health and disease. Trends Immunol. 2019; 40:565-583. doi: 10.1016/j.it.2019.04.012

(26) International Study Group for Behçet's Disease. Criteria for diagnosis of Behçet's disease. Lancet 1990; 335:1078-80.

(27) Perazzio SF, Soeiro-Pereira PV, et al Behçet's disease heterogeneity: cytokine production and oxidative burst of phagocytes are altered in patients with severe manifestations. Clin Exp Rheumatol 2015;33:S85-95.

(28) Emmi G, Becatti M, Bettiol A, et al Behçet's syndrome as a model of thromboinflammation: The role of neutrophils. Front Immunol. 2019; 10:1085. doi: 10.3389/fimmu.2019.01085.

(29) Becatti M, Emmi G, Bettiol A, et al Behçet's syndrome as a tool to dissect the mechanisms of thrombo-inflammation: clinical and pathogenetic aspects. Clin Exp Immunol. 2019;195:322-333. doi: 10.1111/cei.13243. Epub 2018 Dec 18

(30) Johansson $\AA$, Ohlsson S, Pettersson $\AA$, et al Impaired phagocytosis and reactive oxygen species production in phagocytes is associated with systemic vasculitis. Arthritis Research \& Therapy. 2016; 18:18-92.

(31) Elloumi N, Ben Mansour R, Marzouk S, et al Differential reactive oxygen species production of neutrophils_and their oxidative damage in patients with active and inactive systemic lupus erythematosus. Immunol Lett. 2017;184:1-6 doi: 10.1016/j.imlet.2017.01.018

(32) Carmona-Rivera, C. and Kaplan, M. Low-density granulocytes: a distinct class of neutrophils in systemic autoimmunity. Seminars in Immunopathology 2013; 35:455-463. doi: 10.1007/s00281-013-0375-7.

(33) Denny, M., Yalavarthi, S., Zhao, W, et al A distinct subset of proinflammatory neutrophils isolated from patients with systemic lupus erythematosus induces vascular damage and synthesizes type I IFNs. J. Immunol. 2010; 184: 3284-3297. 
(34) Sagiv YJ, Michaeli J, Assi, S., et al Phenotypic diversity and plasticity in circulating neutrophil subpopulations in cancer. Cell Reports 2015; 10: 562-573 doi: 10.1016/j.celrep.2014.12.039

(35) Fresneda Alarcon M, McLaren Z, Wright HL. Neutrophils in the Pathogenesis of Rheumatoid Arthritis and Systemic Lupus Erythematosus: Same Foe Different M.O. Front Immunol. 2021 Mar 4;12:649693. doi: 10.3389/fimmu.2021.649693

(36) Delgado-Rizo V, Martínez-Guzmán, MA, Iñiguez-Gutierrez, L, et al Neutrophil Extracellular Traps and Its Implications in Inflammation: An Overview. Front Immunol. 2017; 8:1-20 doi: 10.3389/fimmu.2017.00081

(37) Hashiba M, Huq A, Tomino A, et al Neutrophil extracellular traps in patients with sepsis. J.Surg. Res. 2015; 194:248-254 doi: 10.1016/j.jss.2014.09.033

(38) Carvalho LO, Aquino EN, Neves AC, Fontes W. The neutrophil nucleus and its role in neutrophilic function. J. Cell Biochem. 2015116 :1831-1836 doi: 10.1002/jcb.25124

(39) Perazzio SF, Soeiro-Pereira PV, Dos Santos VC, et al Soluble CD40L is associated with increased oxidative burst and neutrophil extracellular trap release in Behçet's disease. Arth Res Ther. 2017;19:235. doi: 10.1186/s13075-017-1443-5

(40) Mohanty T, Sjögren J, Kahn F, et al A novel mechanism for NETosis provides antimicrobial defense at the oral mucosa. Blood 2015;126:2128-37. doi: 10.1182/blood2015-04-641142.

(41) Coit P, Mumcu G, Ture-Ozdemir F, et al Sequencing of 165 rRNA reveals a distinct salivary microbiome signature in Behçet's disease. Clin Immunol 2016; 169:28-35. doi: 0.1016/j.clim.2016.06.002

(42) Seoudi N, Bergmeier LA, Drobniewski F, et al The oral mucosal and salivary microbial community of Behçet's syndrome and recurrent aphthous stomatitis. J Oral Microbiol. 2015 Jun 1;7:27150. doi: 10.3402/jom.v7.27150.

(43) Granger V, Faille D, Marani $V$, et al Human blood monocytes are able to form extracellular traps J Leukoc Biol 2017; 102:775-781. doi: 10.1189/jlb.3MA0916-411R.

(44) Nauseef WM, Kubes P. Pondering neutrophil extracellular traps with healthy skepticism. Cell Microbiol 2016; 18:1349-57. doi: 10.1111/cmi.12652

(45) Hemmers S, Teijaro JR, Arandjelovic S, Mowen KA. PAD4-mediated neutrophil extracellular trap formation is not required for immunity against influenza infection. PLoS One. 2011; 6:e22043. doi: 10.1371/journal.pone.0022043.

(46) Pieterse E, Rother N, Yanginlar C, et al Neutrophils Discriminate between Lipopolysaccharides of Different Bacterial Sources and Selectively Release Neutrophil Extracellular Trap Arterioscler Thromb Vasc Biol. 2017 Jul;37(7):1371-1379doi: 10.1161/ATVBAHA.117.309002

(47) Grieshaber-Bouyer R, Nigrovic PA. Neutrophil heterogeneity as therapeutic opportunity in immune-mediated disease. Front Immunol 2019 Mar 4;10:346. doi: 10.3389/fimmu.2019.00346

(48) Wright HL, Lyon M, Chapman EA, Rheumatoid arthritis synovial fluid neutrophils drive inflammation through production of chemokines, reactive oxygen species, and neutrophil extracellular traps. Front Immunol. 2021;11:584116. doi: 10.3389/fimmu.2020.584116 
Figure 1-(A) ROS production in neutrophils of patients with BD $(n=10)$ and healthy controls $(n=17)$. (B) Phagocytic activity in neutrophils of patients with BD $(n=10)$ and controls $(n=16)$. ${ }^{*} \mathrm{P}<0.05,{ }^{* * \mathrm{P}}<0.01, * * * \mathrm{P}<0.001, \mathrm{P}<* * * * 0.0001$, Mann Whitney $\mathrm{U}$ test.

Figure 2-(A) The production of NET by PMA stimulated and unstimulated neutrophils from healthy individuals $(n=10)$ and patients with $B D(n=10)$. (B) The production of NET in E.coli stimulated and unstimulated neutrophils from $\mathrm{HC}(n=10)$ and patients with $B D(n=11)$. (C) The production of NETs in stimulated (with PMA and E.coli) in neutrophil healthy individuals $(n=10)$ and BD patients $(n=1) * P<0.05, * * P<0.01, * * * P<0.001, P<* * * * 0.0001$, Mann Whitney $\mathrm{U}$ test.

Figure 3-The production of NETs in LDN cultures of Healthy individual and BD patients. (A-

D) The isolated cells cultures were seeded into 8 well chamber slides and stimulated with PMA $(25 \mathrm{nM})$ and opsonised E.coli $\left(1-2 \times 10^{9}\right.$ bacteria per $\left.\mathrm{ml}\right)(\mathrm{E}-\mathrm{H})$ and stained with Sytox dye. Images were taken at $\times 20$. Arrow-representing formation of NET (strand of DNA).

Figure 4-The percentage of LDN and NDN in BD, OcMMP patients and healthy individuals. (A) The percentage (count) of $\operatorname{LDN}(n=11)$ and NDN $(n=11)$ in BD patients in comparison to healthy controls $(n=12)$. The percentage of LDN and NDN was investigated based upon the morphology of the isolated cells using Ficol-hypaque gradient. The LDN were isolated from the PBMSC layer and NDN were isolated from the buffy layer on top of red blood cells. $* * * * \mathrm{P}<0.0001$, Mann Whitney U test.

Figure 5-(A) The phagocytic capacity of $\operatorname{LDN}(n=11)$ and NDN in $B D(n=12)$ patients in comparison to healthy controls $(n=12)$. (B)The production of reactive oxygen species by LDN $(n=11)$ and NDN $(n=11)$ in BD patients in comparison to healthy controls $(n=12) .{ }^{*} P<0.05, * * P$ $<0.01, * * * \mathrm{P}<0.001, * * * * \mathrm{P}<0.0001$, Mann Whitney $\mathrm{U}$ test.

Figure 6-(A) The production of NET stimulated with PMA in LDN from healthy controls $(n=10)$ and BD $(n=10)$ patients, unstimulated healthy controls $(n=10)$ and unstimulated BD patients $(n=10)$. (B) The production of NETs in stimulated with E.coli in HC $(n=10)$ and BD $(n=10)$, 
549 unstimulated $\mathrm{HC}(\mathrm{n}=10)$ and unstimulated $\mathrm{BD}(\mathrm{n}=10), \mathrm{HC}(\mathrm{n}=10)$ and stimulated BD patients $550 \quad(\mathrm{n}=10) .{ }^{*} \mathrm{P}<0.05,{ }^{*} \mathrm{P}<0.01, * * * \mathrm{P}<0.001, * * * \mathrm{P}<0.0001$, Mann Whitney $U$ test.

551 Figure 7-(A) The production of NETs stimulated with PMA in NDN from healthy controls $(n=10)$

552 and $B D(n=10)$ patients, unstimulated HC $(n=10)$ and unstimulated BD patients $(n=10)$. (B)

553 The production of NETs in NDN stimulated with E.coli in healthy individuals $(n=10)$ and BD

$554(n=10)$, unstimulated $H C(n=10)$ and unstimulated BD $(n=10)$, stimulated HC $(n=10)$ and

555 stimulated BD patients $(\mathrm{n}=10) .{ }^{*} \mathrm{P}<0.05, * * \mathrm{P}<0.01, * * * \mathrm{P}<0.001, * * * * \mathrm{P}<0.0001$ Mann

556 Whitney U test

557

558 Competing Interests - the authors have no competing interests

559 Funding - we would like to acknowledge funding from The Hale-Rudd Foundation (MM), Fight for

560 Sight (LL) and The Arthur Thompson Trust (MD)

561 Authors contribution - MM processed samples and patient data and carried out the experiments, 562 analysing the data and wrote manuscript; LL and MD processed samples and patient data. GRW, SR

563 and PIM conceived and supervised the project and wrote the manuscript.

564 Acknowledgements - we would like to thank the staff at the Birmingham Centre for Excellence in 565 Behcet's Disease for their support.

566

567

568

569 

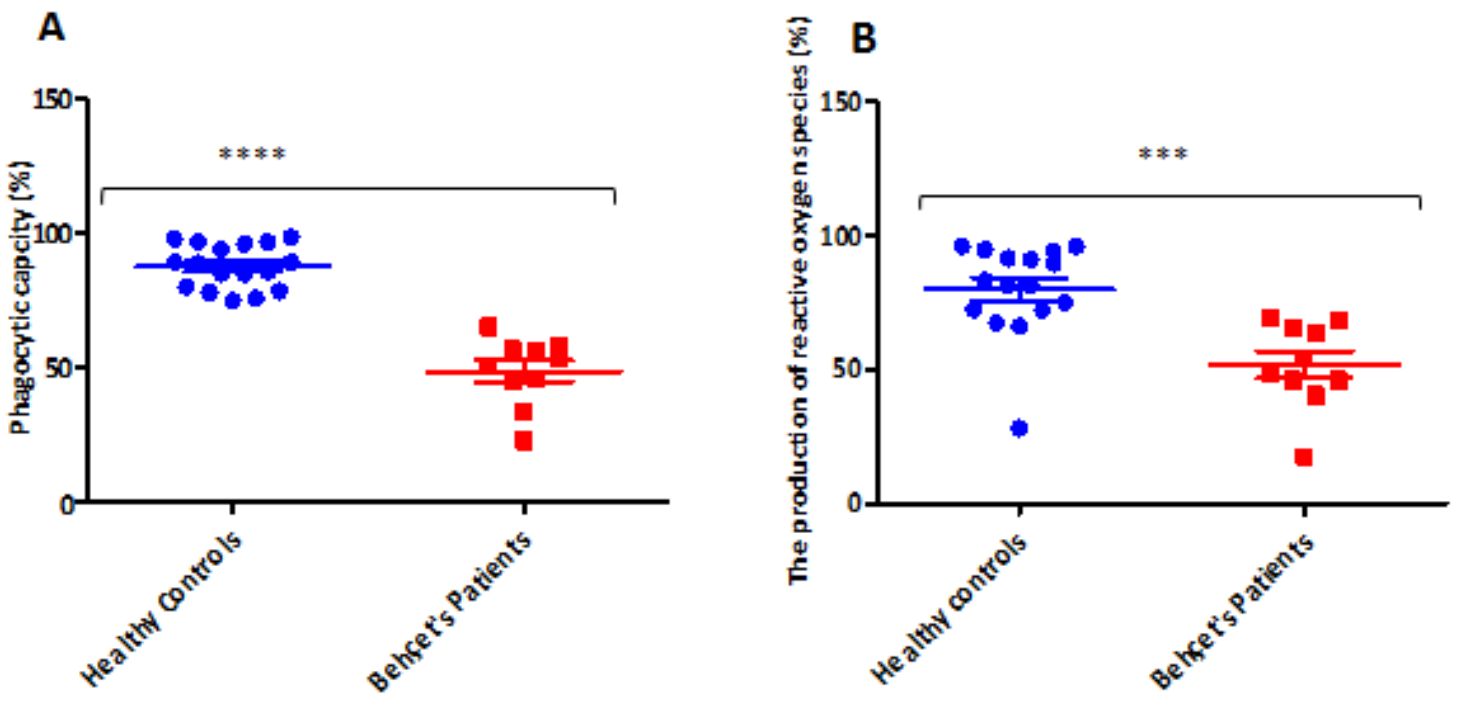

571 


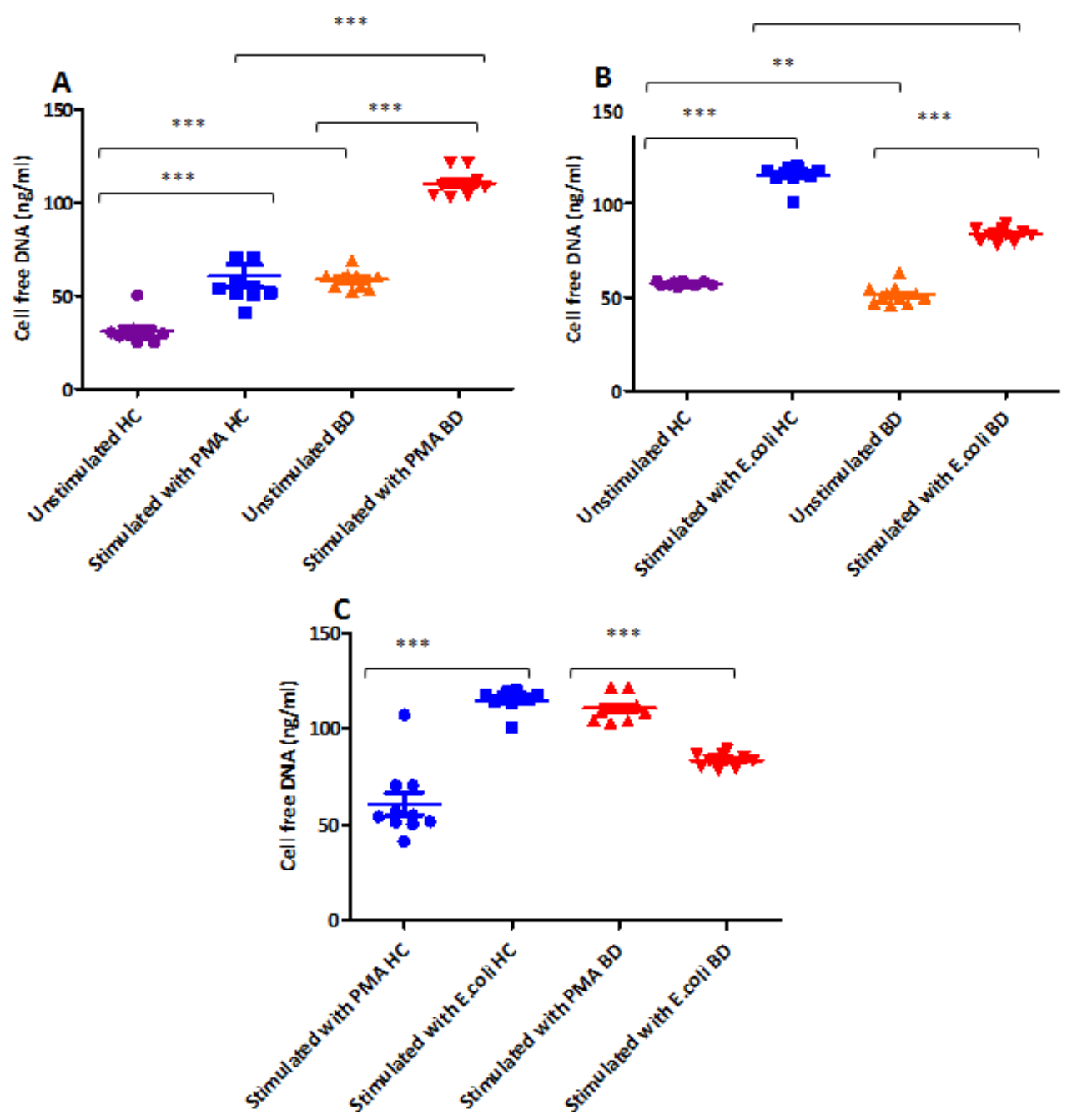

583

584 Figure 2 
587

588

A

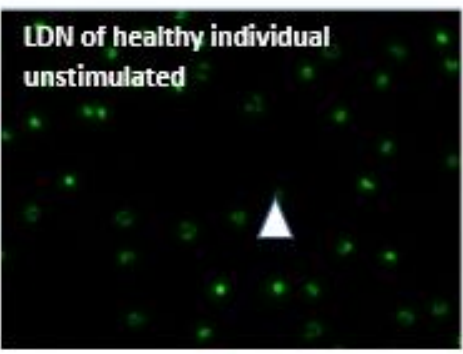

589

590

591

592

593

594

595

596

597

598

599

600

Figure 3

601

\section{B}

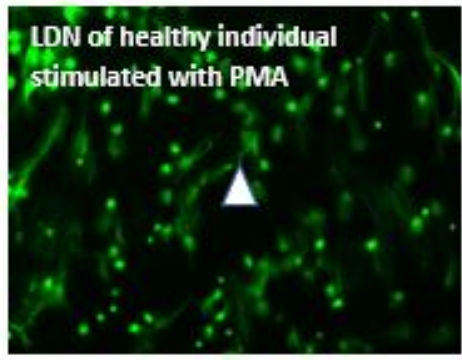

D

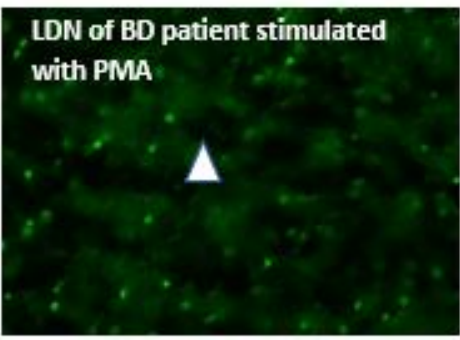

$\mathbf{F}$

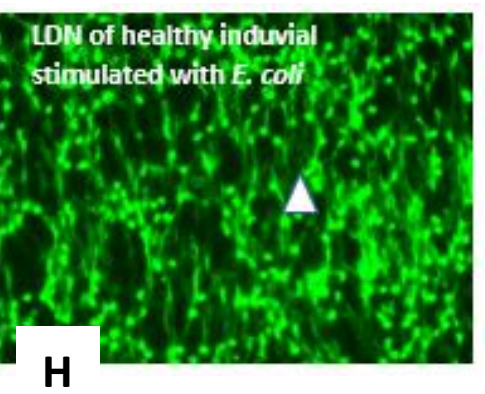

LDN of BD patient stimulated with $E$. coli

$-$

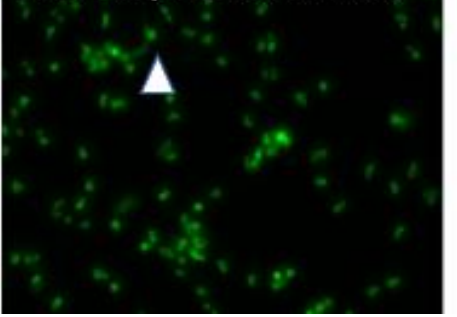

LDN of healthy individual unstimulated

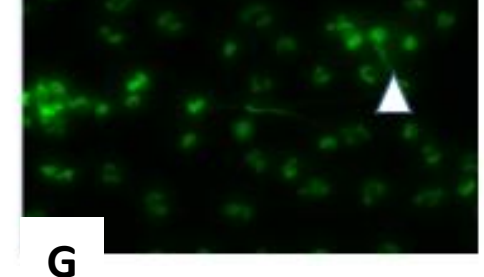

LDN of BD patient unstimulated

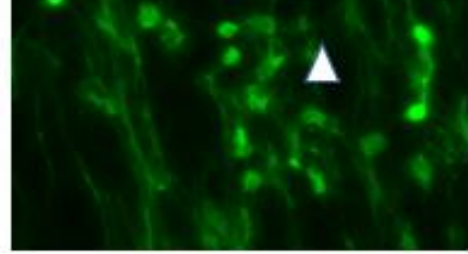




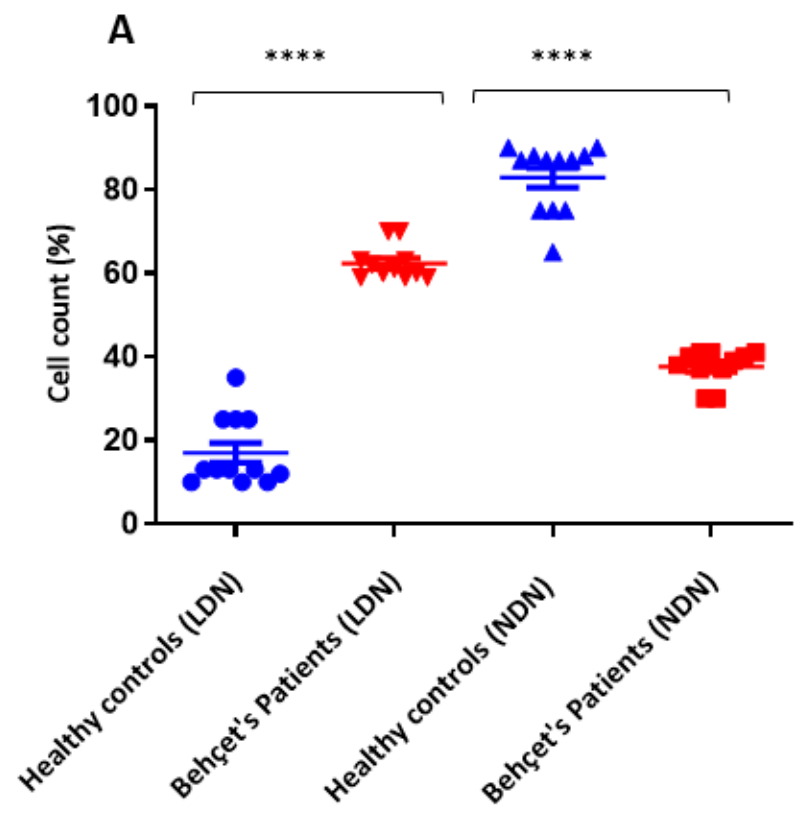

$609 \quad$ Figure 4

610

611

612

613

614 

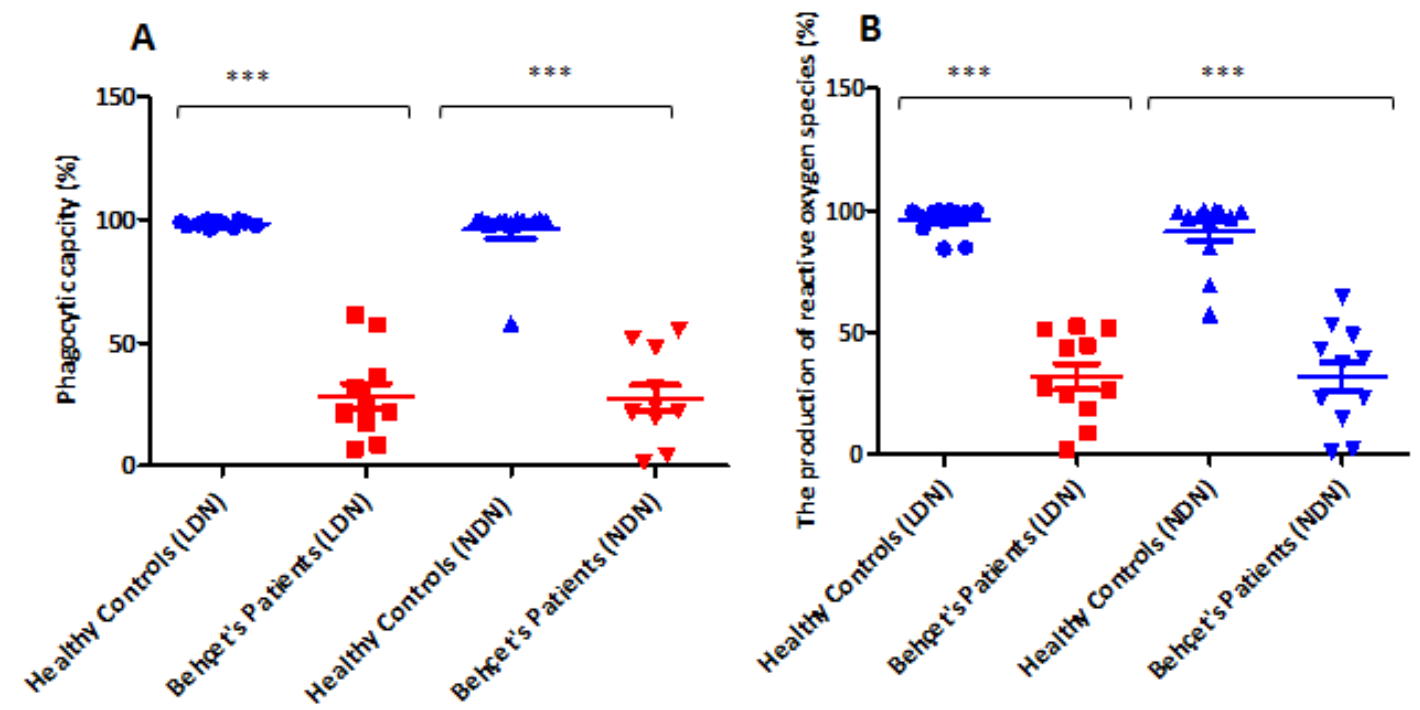

621 Figure 5

622

623

624

625

626

627

628 

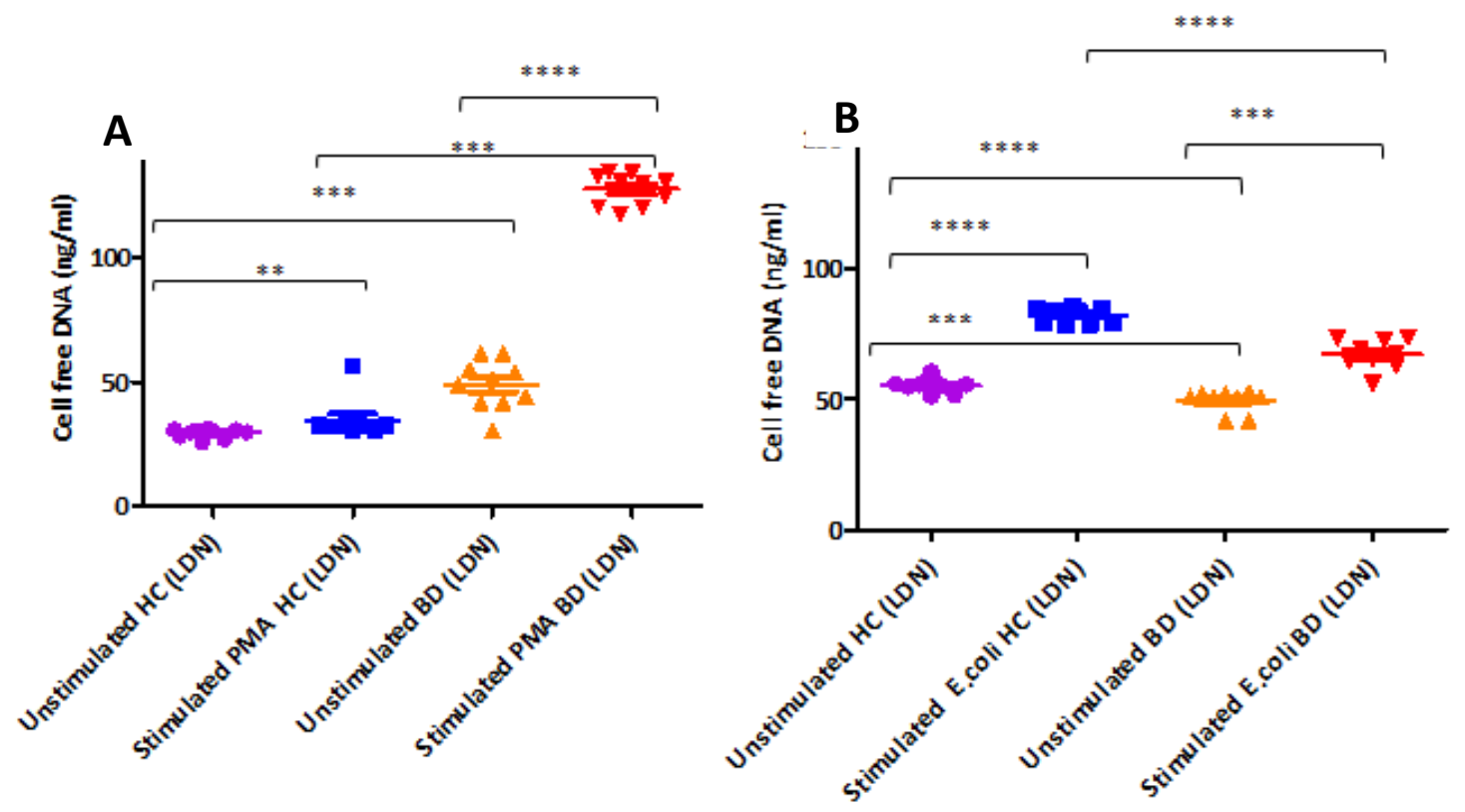

$630 \quad$ Figure 6

631

632

633 

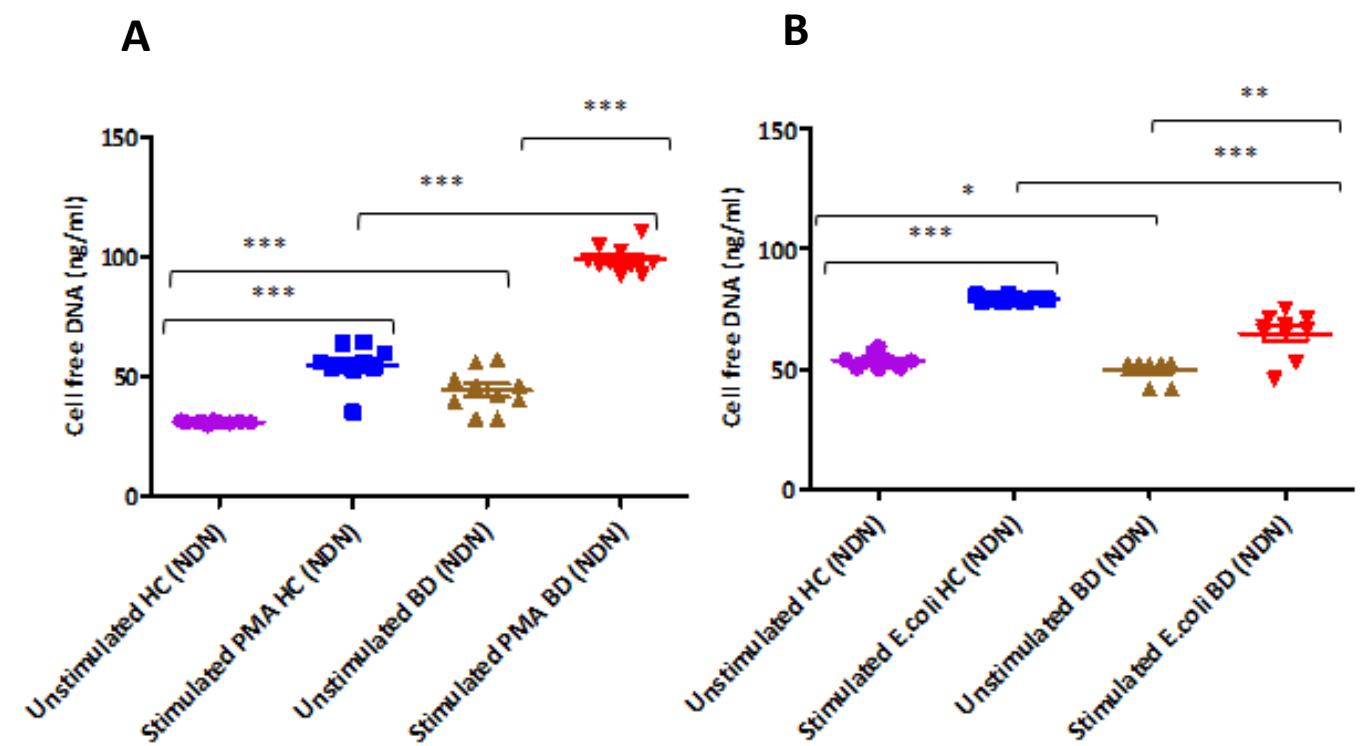

641

$642 \quad$ Figure 7

643

644

645 
Figures
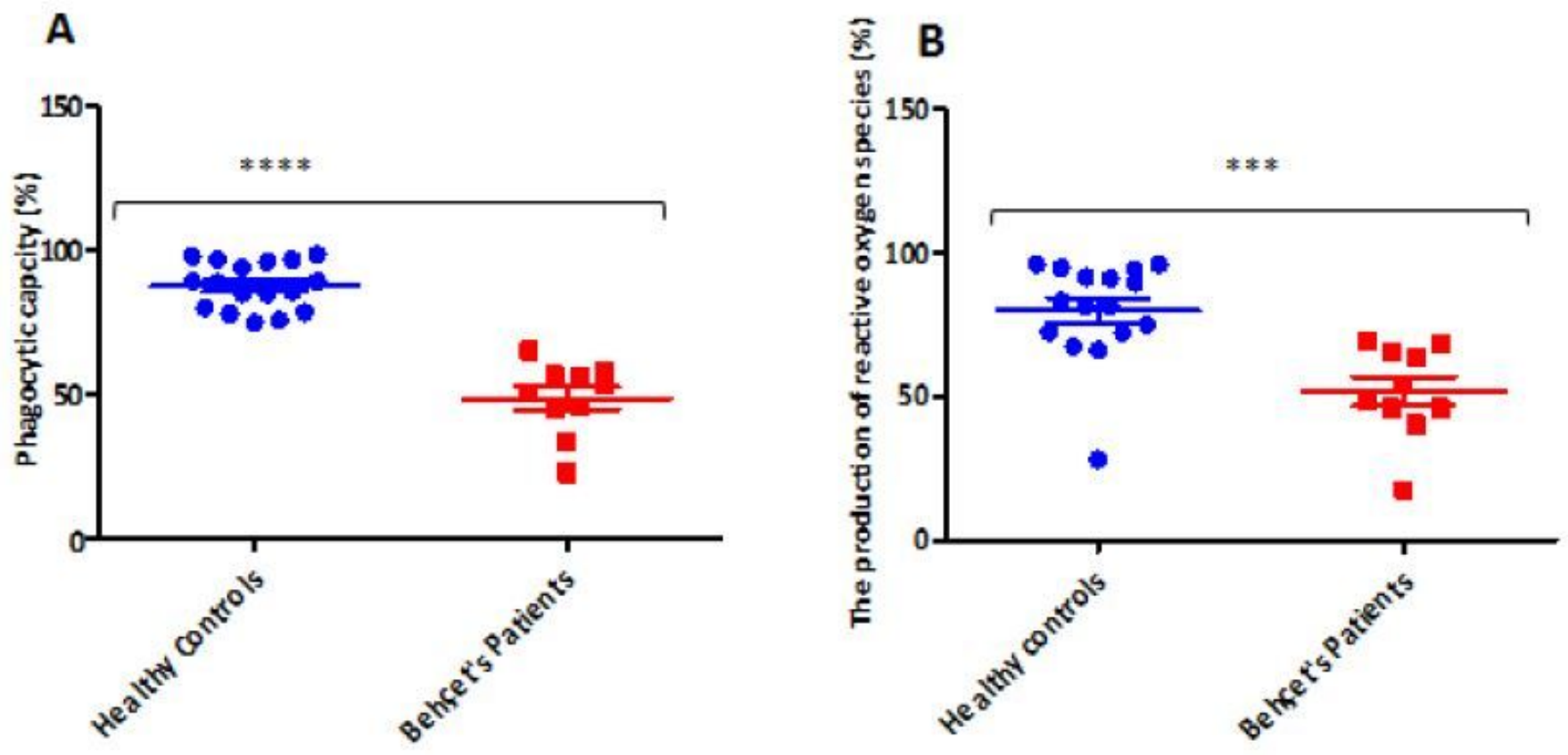

Figure 1

(A) ROS production in neutrophils of patients with BD $(n=10)$ and healthy controls $(n=17)$. (B) Phagocytic activity in neutrophils of patients with $B D(n=10)$ and controls $(n=16) .{ }^{*} P<0.05, \star \star P<<0.01, \star \star \star P<0.001$, $\mathrm{P}<\star \star \star \star 0.0001$, Mann Whitney U test. 

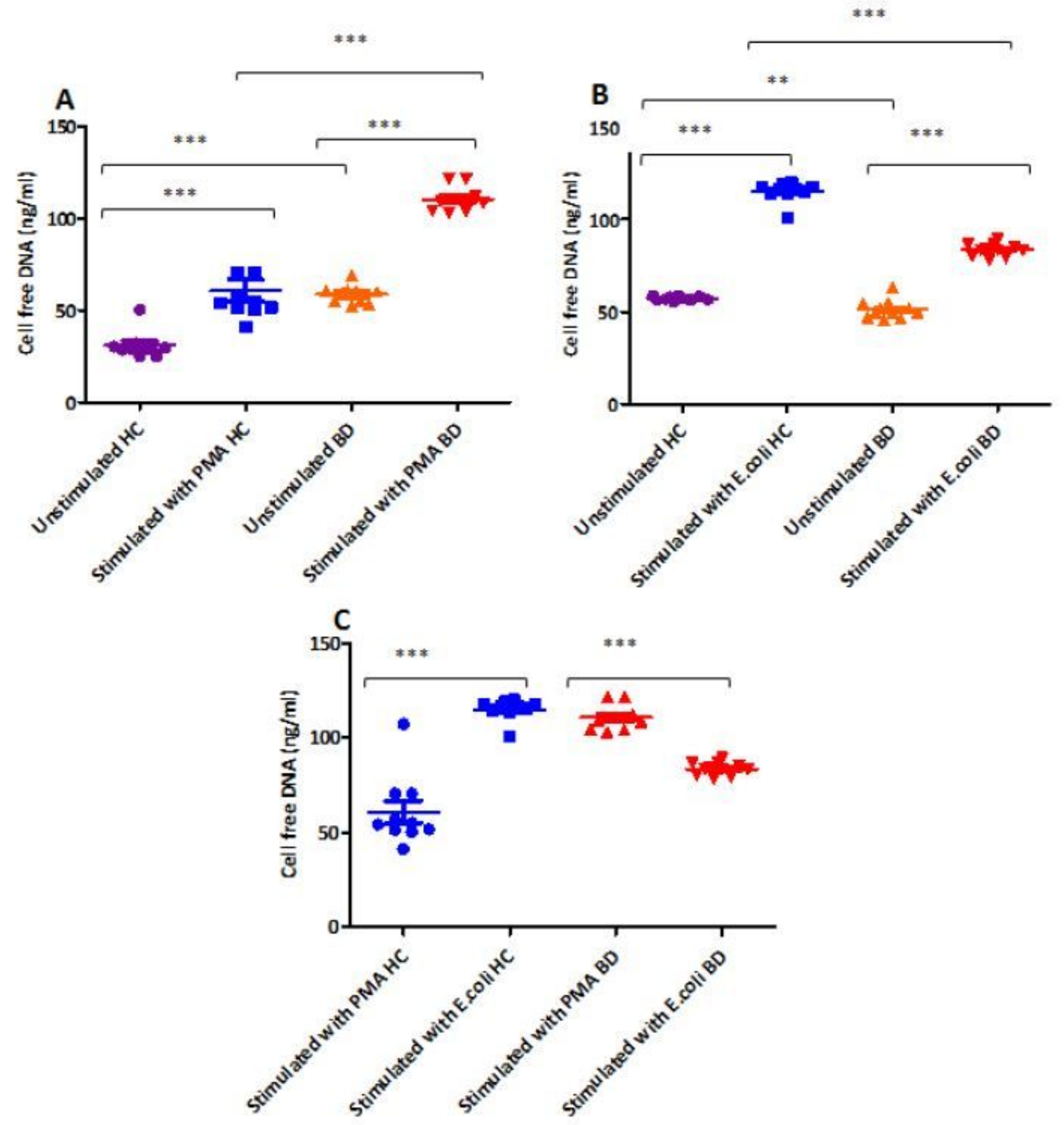

Figure 2

(A) The production of NET by PMA stimulated and unstimulated neutrophils from healthy individuals $(n=10)$ and patients with BD $(n=10)$. (B) The production of NET in E.coli stimulated and unstimulated neutrophils from $\mathrm{HC}(\mathrm{n}=10)$ and patients with $\mathrm{BD}(\mathrm{n}=11)$. (C) The production of NETs in stimulated (with

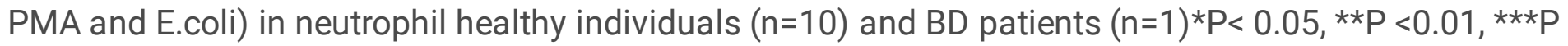
$<0.001, P<\star \star \star \star 0.0001$, Mann Whitney U test. 


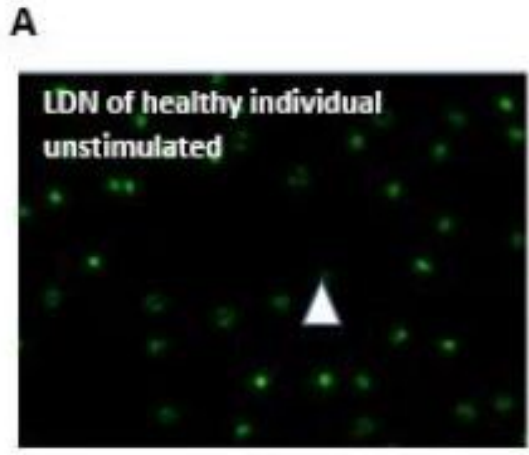

C

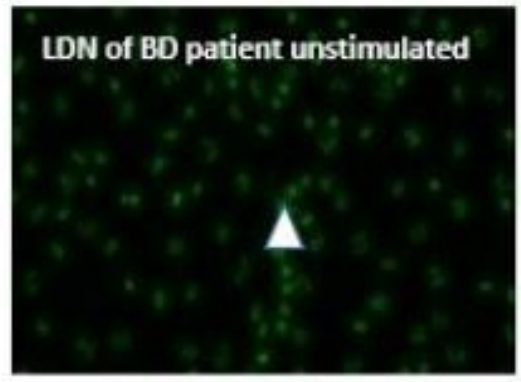

$\mathbf{E}$
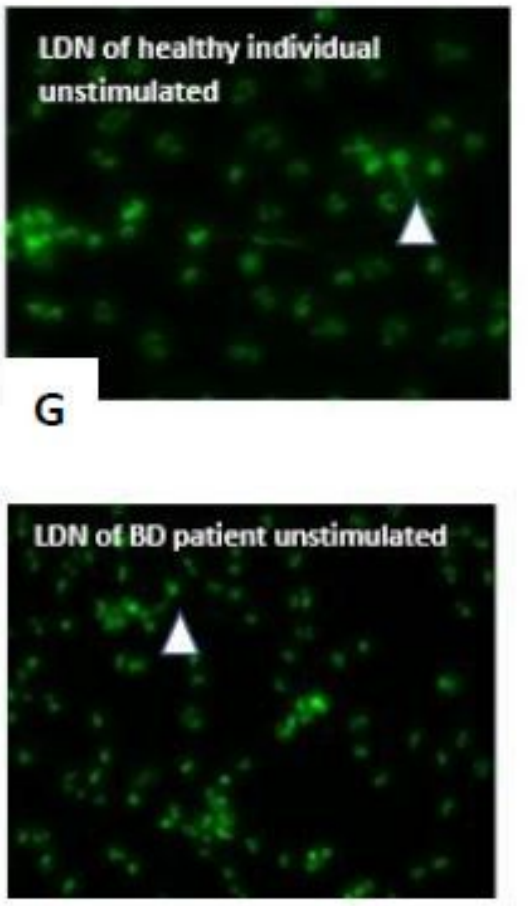

B

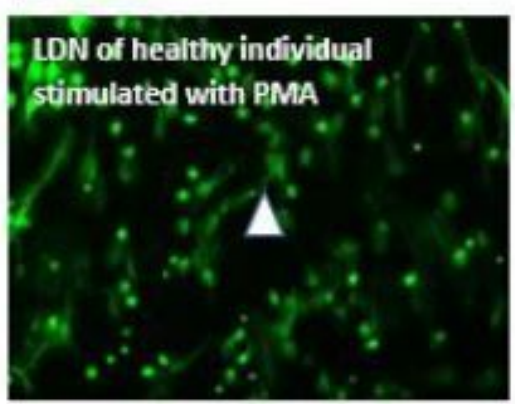

D

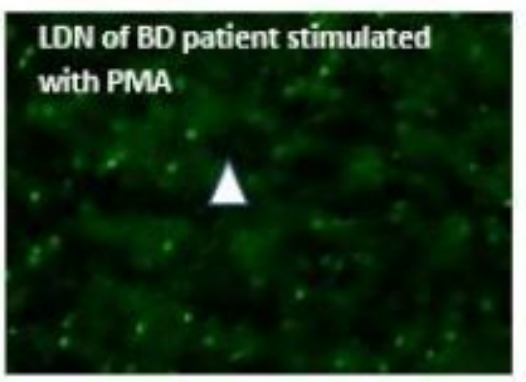

$\mathbf{F}$
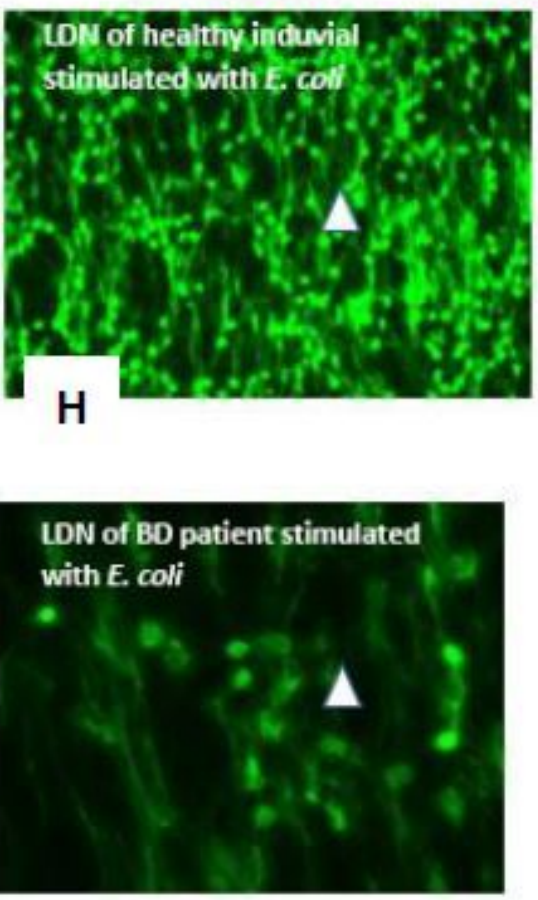

\section{Figure 3}

The production of NETs in LDN cultures of Healthy individual and BD patients. (A-D) The isolated cells cultures were seeded into 8 well chamber slides and stimulated with PMA (25nM) and opsonised E.coli (1-2 $\times 109$ bacteria per $\mathrm{ml})(\mathrm{E}-\mathrm{H})$ and stained with Sytox dye. Images were taken at $\times 20$. Arrowrepresenting formation of NET (strand of DNA). 


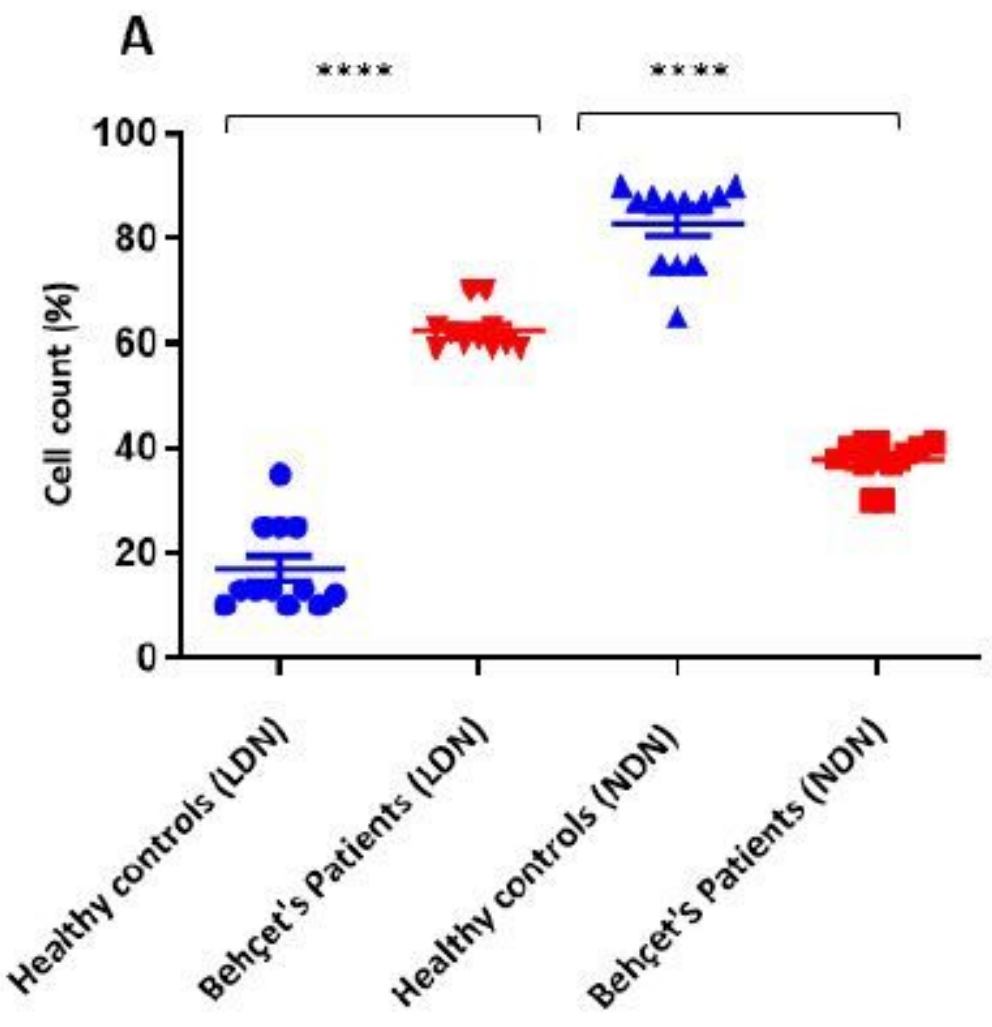

Figure 4

The percentage of LDN and NDN in BD, OcMMP patients and healthy individuals. (A) The percentage (count) of LDN ( $n=11)$ and NDN $(n=11)$ in BD patients in comparison to healthy controls $(n=12)$. The percentage of LDN and NDN was investigated based upon the morphology of the isolated cells using Ficol-hypaque gradient. The LDN were isolated from the PBMSC layer and NDN were isolated from the buffy layer on top of red blood cells. ${ }^{\star * \star *} \mathrm{P}<0.0001$, Mann Whitney $U$ test. 

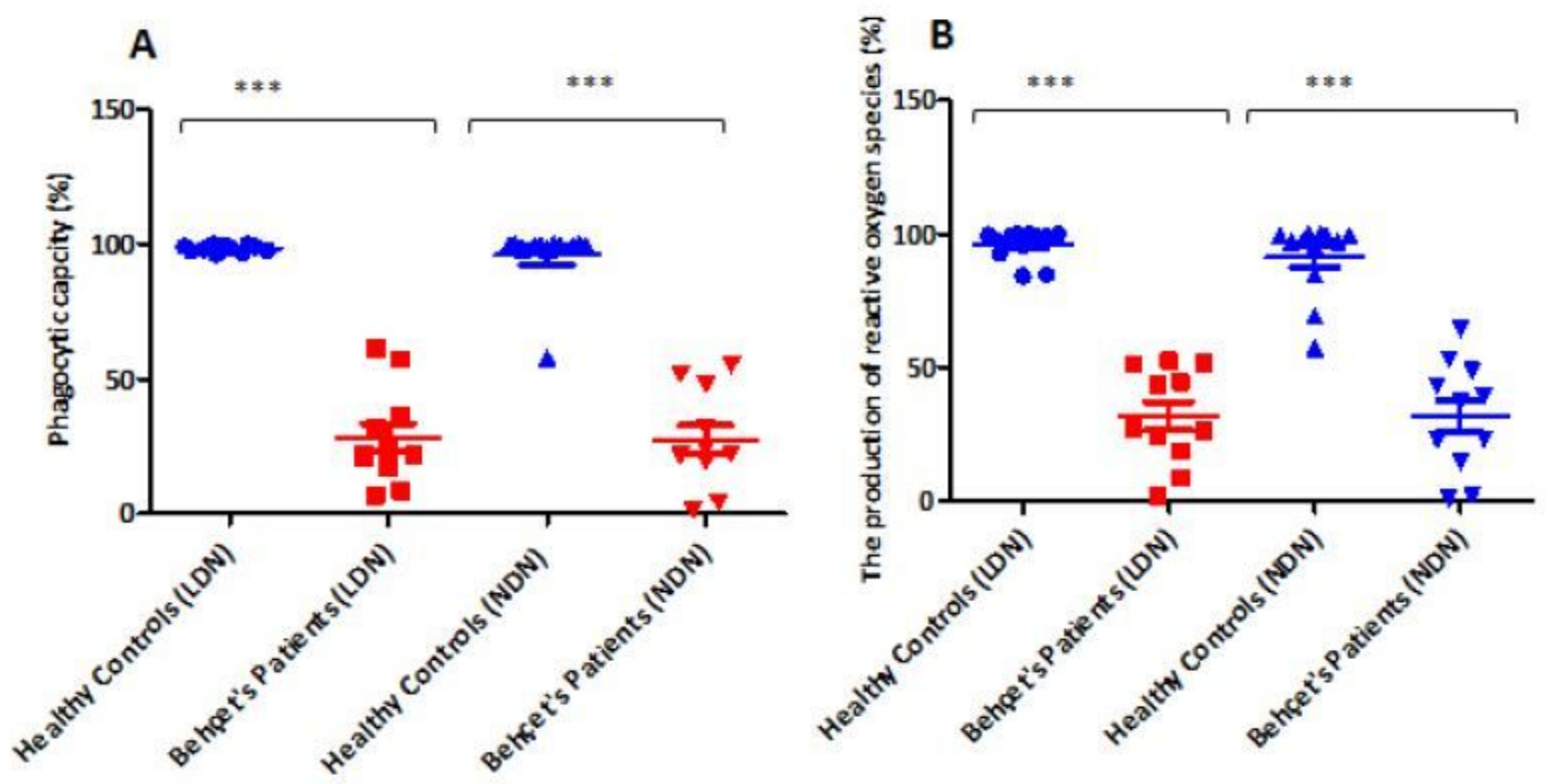

Figure 5

(A) The phagocytic capacity of LDN ( $n=11)$ and NDN in BD ( $n=12)$ patients in comparison to healthy controls $(n=12)$. (B)The production of reactive oxygen species by LDN $(n=11)$ and NDN $(n=11)$ in BD patients in comparison to healthy controls $(n=12) .{ }^{*} P<0.05, * * P<0.01, * \star * P<0.001,{ }^{\star \star * \star} P<0.0001$, Mann Whitney U test.
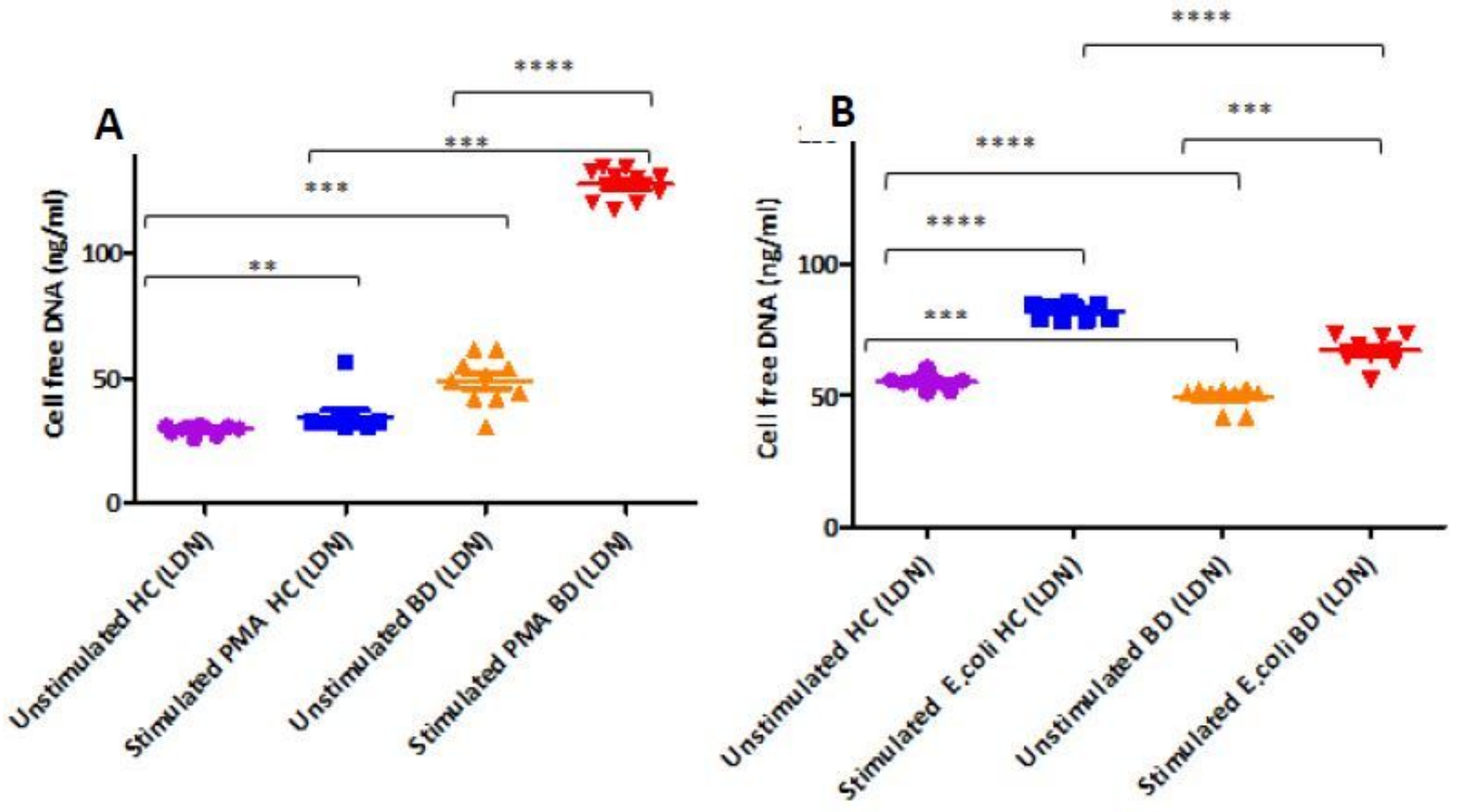
Figure 6

(A) The production of NET stimulated with PMA in LDN from healthy controls $(n=10)$ and BD $(n=10)$ patients, unstimulated healthy controls $(n=10)$ and unstimulated BD patients $(n=10)$. (B) The production of NETs in stimulated with E.coli in HC $(n=10)$ and $B D(n=10)$, unstimulated $\mathrm{HC}(\mathrm{n}=10)$ and unstimulated $B D(n=10), H C(n=10)$ and stimulated BD patients $(n=10) . * P<0.05, * \star P<0.01, * \star * P<0.001, * \star \star \star P<0.0001$, Mann Whitney U test.

A

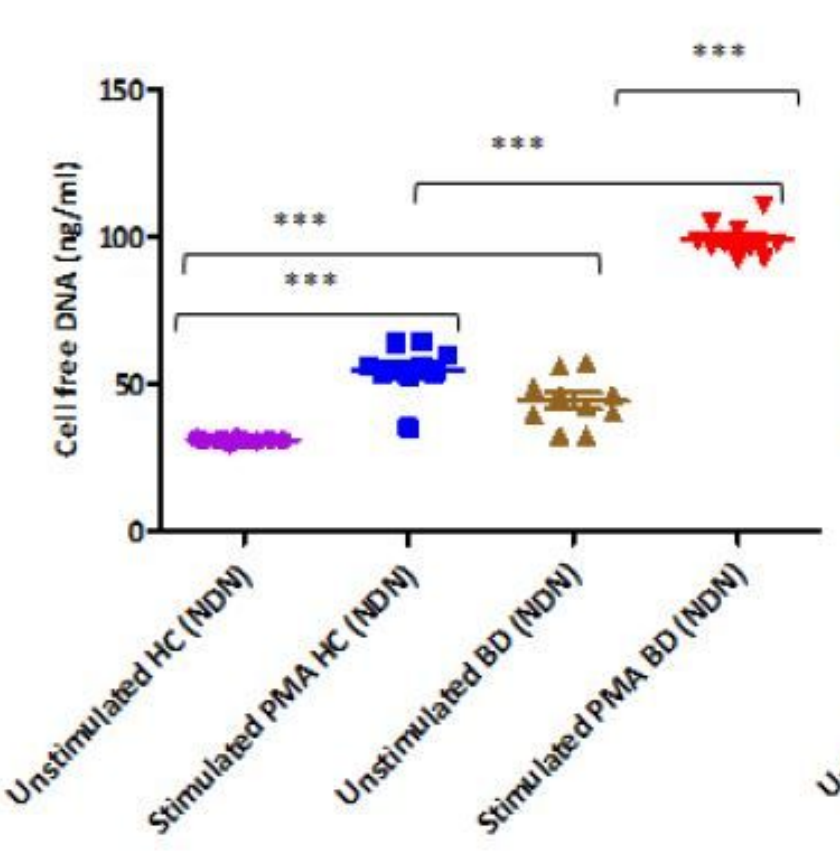

B

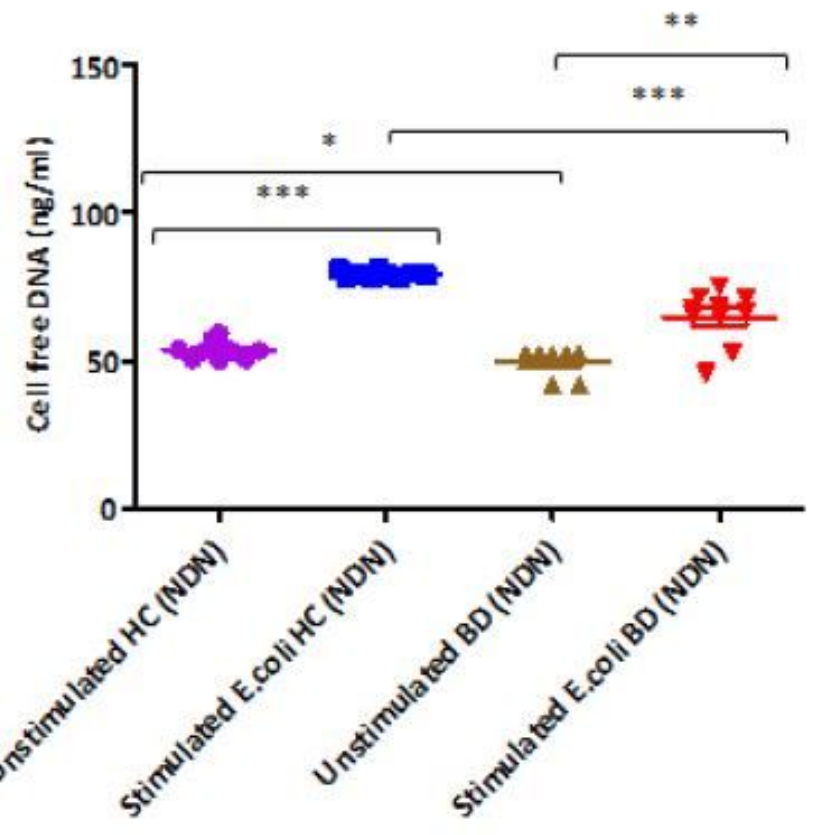

Figure 7

(A) The production of NETs stimulated with PMA in NDN from healthy controls $(n=10)$ and BD $(n=10)$ patients, unstimulated $\mathrm{HC}(\mathrm{n}=10)$ and unstimulated BD patients $(n=10)$. (B) The production of NETs in NDN stimulated with E.coli in healthy individuals $(n=10)$ and $B D(n=10)$, unstimulated $H C(n=10)$ and unstimulated $B D(n=10)$, stimulated $H C(n=10)$ and stimulated $B D$ patients $(n=10) .{ }^{*} P<0.05, * \star P<0.01$, $\star \star \star P<0.001, * \star \star * P<0.0001$ Mann Whitney U test 